\title{
Endoscopic versus surgical approach in the treatment of Zenker's diverticulum: systematic review and meta-analysis
}

Authors

Institutions

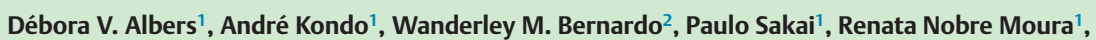
Gustavo Luis Rodela Silva ${ }^{1}$, Edson Ide ${ }^{1}$, Toshiro Tomishige ${ }^{1}$, Eduardo G. H. de Moura ${ }^{1}$

${ }^{1}$ Gastrointestinal Endoscopy Unit, Department of Gastroenterology, Hospital das Clínicas da Faculdade de Medicina da Universidade de São Paulo, Sao Paulo, Brazil

${ }^{2}$ Guidelines Program Coordinator, Brazilian Medical Association, Sao Paulo, Brazil submitted

14. September 2015 accepted after revision 29. March 2016

\section{Bibliography \\ DOI http://dx.doi.org/ \\ 10.1055/s-0042-106203 \\ Published online: 10.5.2016 \\ Endoscopy International Open 2016; 04: E678-E686 \\ (C) Georg Thieme Verlag KG \\ Stuttgart · New York \\ E-ISSN 2196-9736}

\section{Corresponding author}

Débora V. Albers, MD

Hospital das Clinicas Gastroenterology Department Eneas de Carvalho Aguiar Street, 255

Sao Paulo

SP 05403010

Brazil

Fax: +55-11-26616467 deboravieira2001@yahoo.com. br

\section{License terms}

\section{()(1) $\Theta \circledast$}

Background: Zenker's diverticulum is a rare disease in the general population. Its treatment can be carried out by either an endoscopic or surgical approach. The objective of this study was to systematically identify all reports that compare both treatment modalities and to assess the outcomes in terms of length of procedure, length of hospitalization, time until diet introduction, complication rates, and recurrence rates.

Methods: A search of Medline and Embase selected all studies that compared different methods of surgical and endoscopic treatment for Zenker's diverticulum published in the English, Portuguese, and Spanish languages between 1975 and 2014. The meta-analysis was developed in accordance with the Preferred Reporting Items for Systematic Reviews and Meta-analyses (PRISMA) statement. Data were extracted and analyzed for five different outcomes.

Results: Eleven studies met the inclusion criteria, describing outcomes of endoscopic versus surgical treatment for 596 patients with Zenker's di-

\section{Introduction}

$\nabla$

In 1877, Friedrich Albert von Zenker reported the pulsion diverticulum, which was termed Zenker's diverticulum [1]. It is an acquired prolapse of the mucosal and submucosal layers located dorsally at the pharyngoesophageal junction through Killian's triangle [2]. It is the most common type of esophageal diverticula with a reported prevalence ranging between $0.01 \%$ and $0.11 \%$, and typically occurs in middle-aged and elderly patients $[3,4]$. Its pathophysiology in detail remains unknown but it could result from discoordination between pharyngeal contraction and upper esophageal sphincter relaxation [5]. A significant portion of patients with Zenker's diverticulum are asymptomatic [6,7] and in most cases, the condition is diagnosed during a routine evaluation. The most severe complication is the aspiration verticulum. A meta-analysis of the studies suggested a statistically significant reduction in operating time and length of hospitalization, favoring endoscopic treatment (standardized mean difference (SMD)-78.06, 95\%CI-90.63,-65.48 and SMD-3.72, 95\%CI-4.49,-2.95, respectively), just as with the reduction in the fasting period (SMD-4.30, 95\%CI-5.18,-3.42) and risk of complications (SMD-0.09, 95\%CI 0.03, 0.43) for patients who had undergone the endoscopic approach in comparison with the surgical group. Also, a statistically significant reduction in the risk of symptom recurrence was seen when the treatment of Zenker's diverticulum was carried out by a surgical approach compared with endoscopic treatment (SMD 0.08, 95\%CI 0.03, 0.13). Conclusion: Compared with a surgical approach, endoscopic treatment appeared to result in a shorter length of procedure and hospitalization, earlier diet introduction, and lower rates of complications, but in higher rates of symptom recurrence.

of food, especially during the night, which can promote pneumonia. Other rare complications are cancer, bleeding, and perforation [4].

Treatment is indicated for the majority of cases, especially when the patient is symptomatic, regardless of the size of the diverticulum, and consists basically of the myotomy of the cricopharyngeal muscle. Endoscopic treatment was initially proposed in 1917 [8], and developed over time [9-13]. However, potential complications such as perforation, bleeding, and infection have curtailed its use. In 1993, an endoscopic stapling technique using an endosurgical stapler was presented that, at the same time, cut the septum of the pouch and stapled the wound edges closed [14]. The techniques in the surgical approach were cricopharyngeal myotomy and suspension of pouch; cricopharyngeal myotomy and inversion of pouch; cricopharyngeal myotomy and ex- 


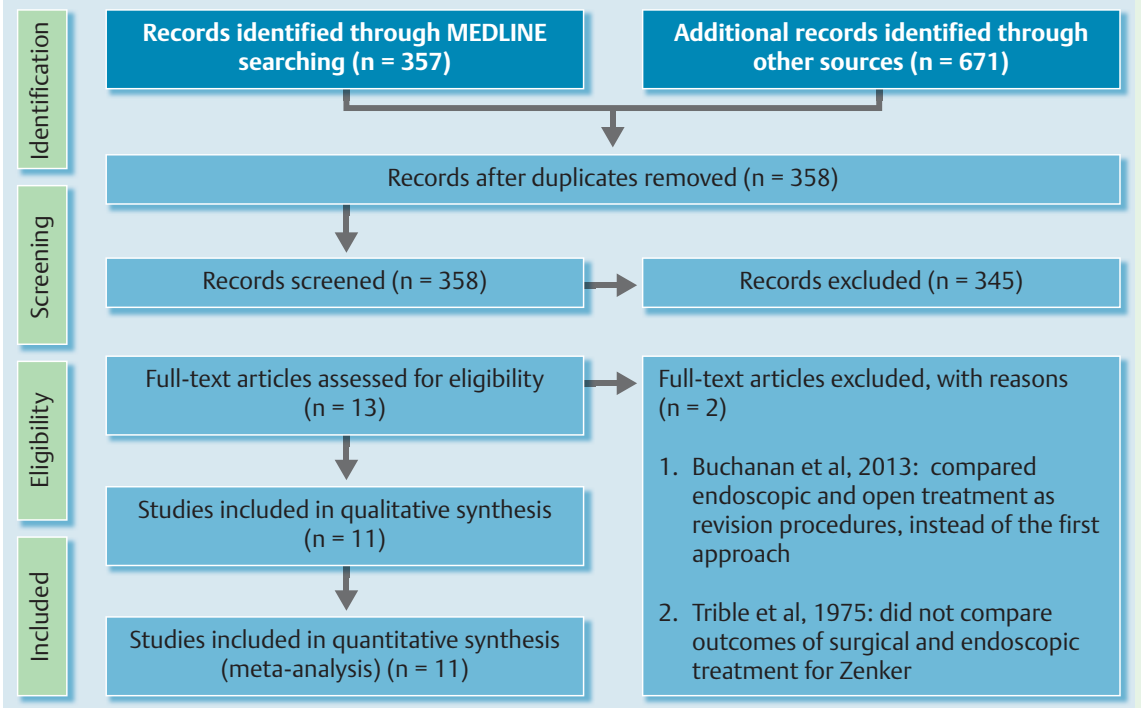

Fig. 1 PRISMA flow diagram providing information on the selection of studies.

cision of pouch; cricopharyngeal myotomy only; Dolman's procedure and pouch excision only [15].

The aims of this systematic review were to identify all published studies that compared endoscopic versus surgical treatment of Zenker's diverticulum and assess outcomes in terms of length of procedure, length of hospitalization, time of diet introduction, rates of complication, and rates of recurrence.

\section{Methods}

The review was registered on the PROSPERO international database (www.crd.york.ac.uk/prospero/), number CRD42014014675.

\section{Data search}

A systematic review of articles reporting endoscopic versus surgical treatment of Zenker's diverticulum was conducted in accordance with the Preferred Reporting Items for Systematic Reviews and Meta-Analyses (PRISMA) guidelines.

Studies have been selected by searching electronic databases and scanning reference lists of articles. This search was applied for Medline (considering all years) and Embase (considering all years). Cochrane and LILACS (via BVS), Scopus and CINAHL (via
EBSCO) databases were also reviewed. The last search was run on 18 September 2014 and no limits were applied for language. The following search strategy was used for Medline database, named \#1 for abbreviation purposes and stratified by Population $(\mathrm{P})$, Intervention $(\mathrm{I})$, and Comparison $(\mathrm{C})$ :

P: Zenker (MeSH) AND

I: [Endoscopy (MeSH) OR Endoscopy, Digestive System (MeSH) OR Endoscopy, Gastrointestinal (MeSH)] AND

C: [Surgical Procedures, Operative (MeSH) OR Digestive System Surgical Procedures (MeSH) OR Surgery (Subheading)].

Aiming to select high quality studies, the search strategy mentioned above (\#1) was filtered twice, as follows: (a) “(\#1 AND random *)", called \#2; and (b) ((clinical[Title/Abstract] AND trial [Title/Abstract]) OR clinical trials as topic [MeSH Terms] OR clinical trial[Publication Type] OR random*[Title/Abstract] OR random allocation[MeSH Terms] OR therapeutic use [MeSH Subheading]), called \#3. The totality of articles was obtained by mixing the investigation in the PubMed Advanced Search Builder as follows: "(\#2 OR \#3)". As part of the process, the Medline search strategies were peer reviewed.

For Embase, Cochrane, LILACS, Scopus, and CINAHL databases, the search was: "(Zenker's diverticulum AND endoscopy AND surgery)".

Table 1 Summary of the characteristics of the studies that met the inclusion criteria.

\begin{tabular}{|c|c|c|c|c|c|c|c|}
\hline \multirow[t]{2}{*}{ Study } & \multicolumn{3}{|c|}{ No. of patients } & \multicolumn{3}{|l|}{ Measures } & \multirow[t]{2}{*}{ Outcomes } \\
\hline & Total & Endoscopy & Surgery & Method & Age & Size & \\
\hline Seth et al., 2014 [18] & 55 & 24 & 31 & Stapler & $\mathrm{Y}$ & $\mathrm{Y}$ & LP, M, COMP, REC, IS, CR, LOSS \\
\hline Henry et al., 2013 [2] & 36 & 12 & 24 & Stapler & $\mathrm{Y}$ & Y & M, COMP, REC, IS, CR \\
\hline Koch et al., 2011 [19] & 155 & 101 & 54 & Laser & $\mathrm{Y}$ & N & LP, LH, COMP, IS, CR, LOSS \\
\hline Brace et al., 2010 [20] & 18 & 10 & 8 & Stapler & Y & Y & LP, LH, COMP, LOSS \\
\hline Porcuna et al., 2009 [21] & 16 & 6 & 10 & Combined & Y & Y & LP, DI, M, COMP, REC, CR \\
\hline Wirth et al., 2006 [22] & 47 & 23 & 24 & Stapler & Y & $\mathrm{N}$ & LP, LH, M, COMP, LOSS \\
\hline Safdar et al., 2004 [23] & 19 & 10 & 9 & Stapler & Y & N & LP, LH, DI, COMP, REC \\
\hline Smith et al., 2002 [24] & 16 & 8 & 8 & Stapler & $\mathrm{N}$ & N & LP, LH, DI, COST \\
\hline Gutschow et al., 2002 [25] & 184 & 86 & 98 & Both singly & Y & Y & LH, DI, COMP \\
\hline Sydow et al, 2001 [26] & 16 & 3 & 13 & Both singly & Y & Y & COMP, REC \\
\hline van Eeden et al., 1999 [15] & 34 & 17 & 17 & Stapler & $\mathrm{Y}$ & N & LP, LH, DI, COMP, REC, IS, LOSS \\
\hline
\end{tabular}

Y: information present; N: missing information; LP: length of procedure; LH: length of hospitalization; DI: time to diet introduction; M: mortality; COMP: complications; REC: recurrence; IS: improvement in symptoms; CR: conversion rates; LOSS: loss on follow-up; COST: costs. 
Table 2 Newcastle-Ottawa Scale; all studies had acceptable risk of bias.

\begin{tabular}{|c|c|c|c|c|c|c|c|}
\hline \multirow{2}{*}{$\begin{array}{l}\text { Study } \\
\text { Seth et al., } 2014 \text { [18] }\end{array}$} & \multicolumn{3}{|c|}{ Selection } & Comparability & \multicolumn{2}{|c|}{ Exposure } & \multirow{2}{*}{$\begin{array}{l}\text { Total score } \\
8\end{array}$} \\
\hline & * * & * & * & $*$ & * & * & \\
\hline Henry et al., 2012 [2] & $*$ & * & * & * & * & * & 8 \\
\hline Koch et al., 2011 [19] & * & * & * & $*$ & * & * & 8 \\
\hline Brace et al., 2010 [20] & * & * & & * & * & * & 8 \\
\hline Porcuna et al., 2009 [21] & * & * & $*$ & * & * & * & 9 \\
\hline Wirth et al., 2006 [22] & * & * & * & * & * & * & 8 \\
\hline Safdar et al., 2004 [23] & * & * & * & * & * & * & 9 \\
\hline Smith et al., 2002 [24] & * & * & * & $*$ & * & * & 9 \\
\hline Gutschow et al., 2002 [25] & * & * & * & * & * & * & 9 \\
\hline Sydow et al., 2001 [26] & * & * & * & * & * & * & 8 \\
\hline van Eeden et al., 1999 [15] & $*$ & * & * & * & * & * & 9 \\
\hline
\end{tabular}

Included trials should have a score $\geq 6$, with a total of 9 being the highest possible score (each asterisk symbol represents one point).

Table 3 Results of all 11 studies summarized.

\begin{tabular}{|c|c|c|c|c|c|c|c|c|}
\hline Study & $\begin{array}{l}\text { Total, } \\
\text { n }\end{array}$ & $\begin{array}{l}\text { Endoscopy, } \\
\text { n }\end{array}$ & $\begin{array}{l}\text { Sur- } \\
\text { gery, n }\end{array}$ & $\begin{array}{l}\text { Length of proce- } \\
\text { dure, } \min \end{array}$ & $\begin{array}{l}\text { Length of hospi- } \\
\text { talization, days }\end{array}$ & $\begin{array}{l}\text { Time to diet intro- } \\
\text { duction, days }\end{array}$ & $\begin{array}{l}\text { Compli- } \\
\text { cation }\end{array}$ & $\begin{array}{l}\text { Recur- } \\
\text { rence }\end{array}$ \\
\hline Seth et al., 2014 [18] & 55 & 24 & 31 & $48.3 / 131.1$ & IU & IU & $7 / 2$ & $8 / 3$ \\
\hline Henry et al., 2012 [2] & 36 & 12 & 24 & IU & IU & IU & $0 / 4$ & $4 / 0$ \\
\hline Koch et al., 2011 [19] & 155 & 101 & 54 & $51 / 146$ & $8.7 / 10.4$ & IU & $9 / 14$ & $12 / 1$ \\
\hline Brace et al., 2010 [20] & 18 & 10 & 8 & $19.5 / 110.88$ & $2.3 / 4.71$ & $1.1 / 2$ & $1 / 0$ & $0 / 0$ \\
\hline Porcuna et al., 2009 [21] & 16 & 6 & 10 & $45 / 90$ & $2 / 3.8$ & $1 / 1.5$ & $0 / 2$ & $0 / 2$ \\
\hline Wirth et al., 2006 [22] & 47 & 23 & 24 & $32 / 106$ & $5.5 / 12.3$ & IU & $3 / 4$ & $1 / 1$ \\
\hline Safdar et al., 2004 [23] & 19 & 10 & 9 & $20-30 / 90-120$ & $3.9 / 10$ & $1 / 6$ & $1 / 3$ & $1 / 2$ \\
\hline Smith et al., 2002 [24] & 16 & 8 & 8 & $25.5 / 87.6$ & $1.3 / 5.2$ & $0.8 / 5.1$ & $1 / 0$ & $0 / 0$ \\
\hline Gutschow et al., 2002 [25] & 184 & 86 & 98 & IU & $4 / 6.12$ & $2 / 4$ & $3 / 3$ & $11 / 8$ \\
\hline Sydow et al., 2001 [26] & 16 & 3 & 13 & IU & IU & IU & $0 / 3$ & $2 / 2$ \\
\hline van Eeden et al., 1999 [15] & 34 & 17 & 17 & IU & $2.26 / 4$ & $3 / 3.6$ & $3 / 7$ & $0 / 0$ \\
\hline
\end{tabular}

IU: information unavailable.

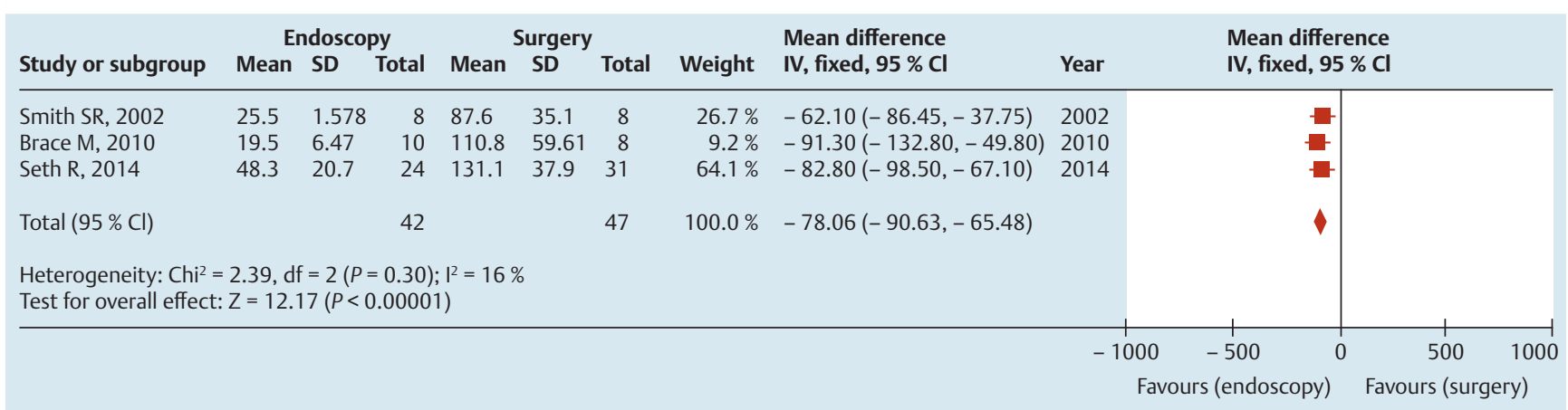

Fig. 2 Length of procedure: endoscopic and surgical treatment of Zenker's diverticulum-forest plot.

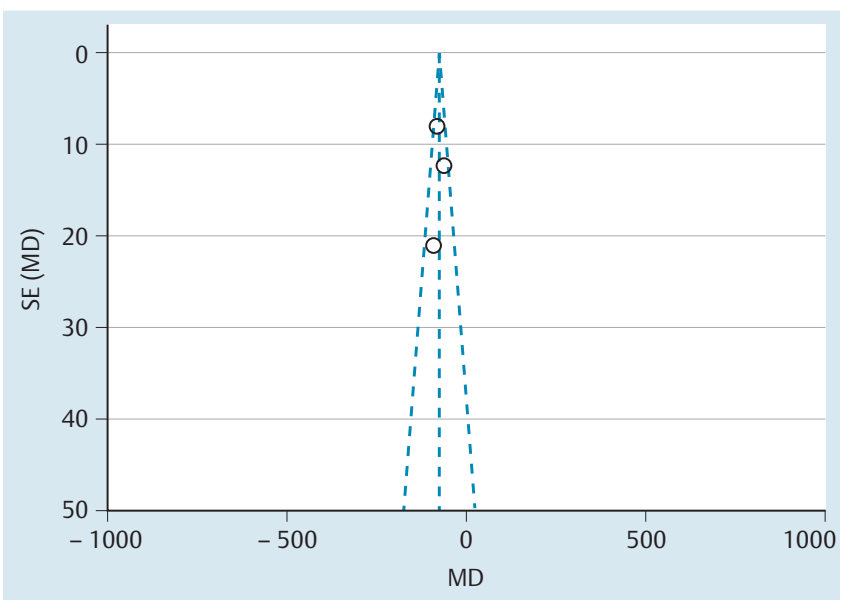

Fig. 3 Length of procedure: endoscopic and surgical treatment of Zenker's diverticulum-funnel plot.

\section{Selection criteria}

Clinical trials and/or observational studies were initially identified and targeted to the selection process. No limits on language or publication data were initially imposed. The titles and abstracts of all potentially relevant studies were reviewed independently by two authors for eligibility. Disagreements between the reviewers were resolved by consensus. The references in the included studies were then selected for any relevant articles.

Studies that compared endoscopic versus surgical treatment of Zenker's diverticulum were included. There were no restrictions with regard to different modalities of treatment in each arm. Studies were excluded if they did not compare both techniques or if different techniques were combined in the same patient. We also excluded studies that were written in languages different from English, Portuguese or Spanish, as well as reviews and 


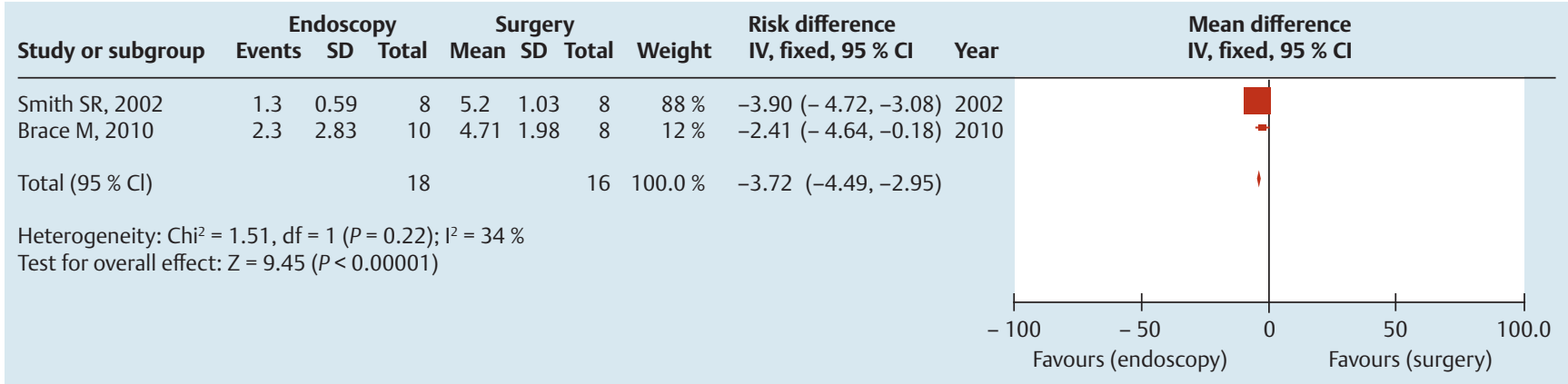

Fig. 4 Length of hospitalization: endoscopic and surgical treatment of Zenker's diverticulum-forest plot.

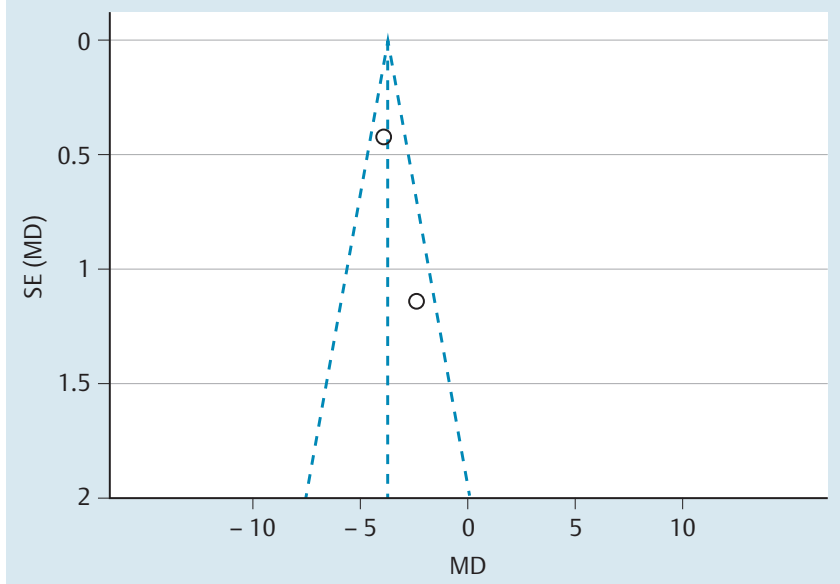

Fig. 5 Length of hospitalization: endoscopic and surgical treatment of Zenker's diverticulum-funnel plot.

case reports. There were no exclusions based on patient numbers or length of follow-up.

The main outcome measures were length of procedure, length of hospitalization, time until diet introduction, rate of complications, and rate of recurrences. To summarize the study selection process, an adapted PRISMA Flow Diagram was used [16].

\section{Data extraction}

Details, such as number of patients, age, gender, method of treatment, size of the diverticulum, and study design were recorded from all included articles when available.

The modality of intervention was also assessed. Any disagreements in the process of study selection and data extraction were resolved by discussion and consensus.

\section{Risk of bias in individual studies}

The articles were analyzed for selection bias, quality bias, publication bias, and sensitivity analysis. To avoid selection bias, studies were selected after extensive searching in electronic databases and scanning reference lists of articles that applied to the inclusion criteria. This search was applied for Medline, Embase, LILACS, Scopus, and CINAHL databases. To prevent quality bias, the Newcastle-Ottawa Scale [17] was applied. The studies selected were all assessed if the risk of bias was acceptable, i.e., critical evaluation of the included trials should reveal a score $\geq 6$, with a total of 9 being the highest possible score.

\section{Summary measures and synthesis of results}

Heterogeneity between studies was assessed using the I2 index introduced by Higgins and Thompson [27,28]. For computations of confidence intervals, estimates of mean and standard deviation (mean \pm SD) were required. However, some of the published articles did not describe the mean and standard deviation, and did not show data that would enable calculation, so they were not included in the meta-analysis. Funnel plots were produced to identify the presence of publication bias in the meta-analysis. Both total sample size and precision (1/standard error) were plotted against the treatment effects (absolute risk, absolute risk increase, and absolute risk reduction for binary variables and standardized mean difference (SMD) for continuous variables) [29]. The outcome was considered to be statistically significant if the confidence interval of $95 \%$ was achieved.

The I 2 statistic is appealing because it may be expressed as a percentage, and this percentage has an intuitive meaning. It represents the percentage of variation in the data that is due to between-study variability. Thus, a perfectly homogeneous study will have an I2 value of 0 . Conversely, a perfectly heterogeneous set of studies would have an I2 value approaching 100\% [30].

The statistical method used for the meta-analysis was the fixed effects model. A fixed-effects model makes the presupposition that each of the studies in the meta-analysis was studying the same overall population of patients. Therefore, it only takes into account the variation within studies.

Depending on heterogeneity, as measured by I2, data were calculated by the fixed effects model using the Mantel-Haenszel method [31]. When the heterogeneity was higher than $50 \%$, sensitivity analysis was performed, generating a new analysis. Subgroup analyses and meta-regression were performed to identify the origin of heterogeneity. All analyses were performed using Review Manager 5.3 Software (Cochrane Informatics \& Knowledge Management Department) [32].

\section{Results}

\section{$\nabla$}

\section{Study selection}

The process of study selection is summarized in 0 Fig. 1. During the systematic review, 357 articles were identified through MEDLINE searching. Another 671 were screened in EMBASE, CINAHL, LILACS, and BVS databases. One additional study was not identified in MEDLINE, giving a total of 358 articles. Of those, 345 were excluded for different reasons (did not meet the inclusion criteria, language, systematic reviews, and case reports). After the abstracts had been carefully analyzed, 13 articles were assessed for eligibility. Among them, only 11 studies met the inclusion crite- 


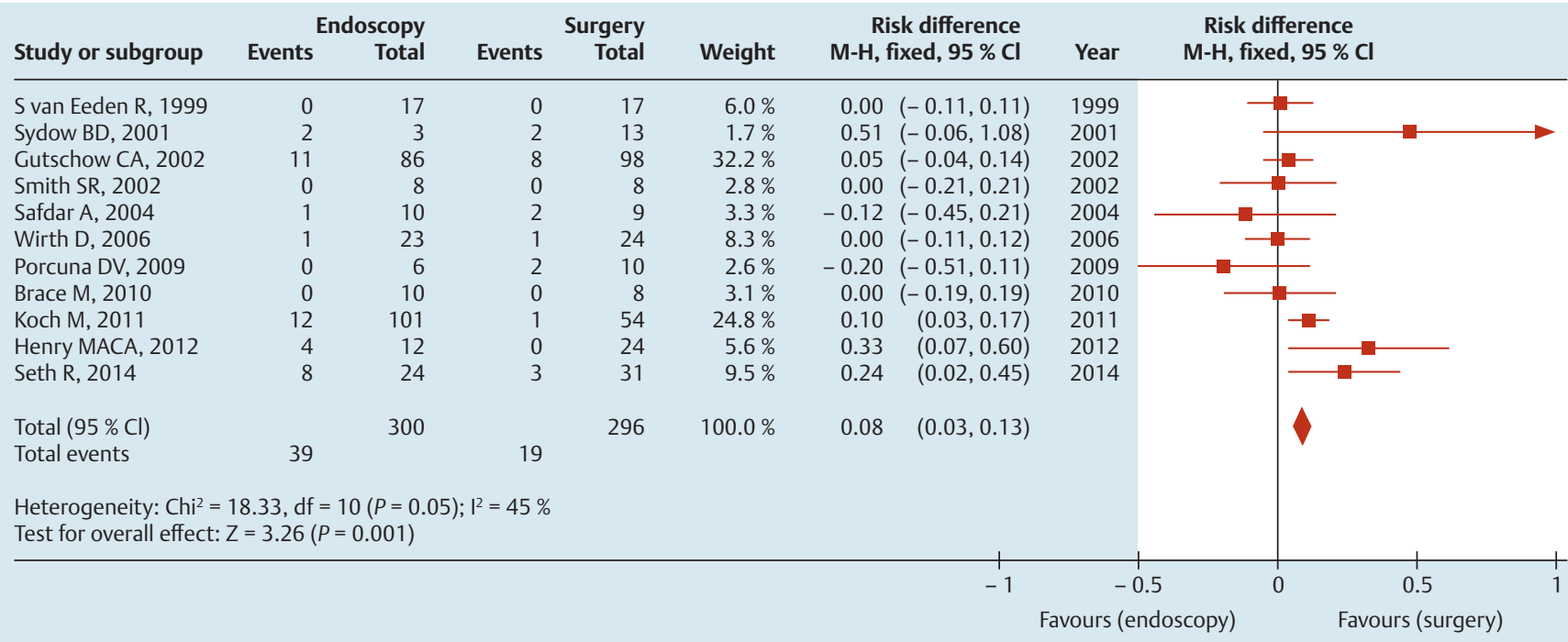

Fig. 6 Recurrence of Zenker's diverticulum: endoscopic and surgical treatment-forest plot.

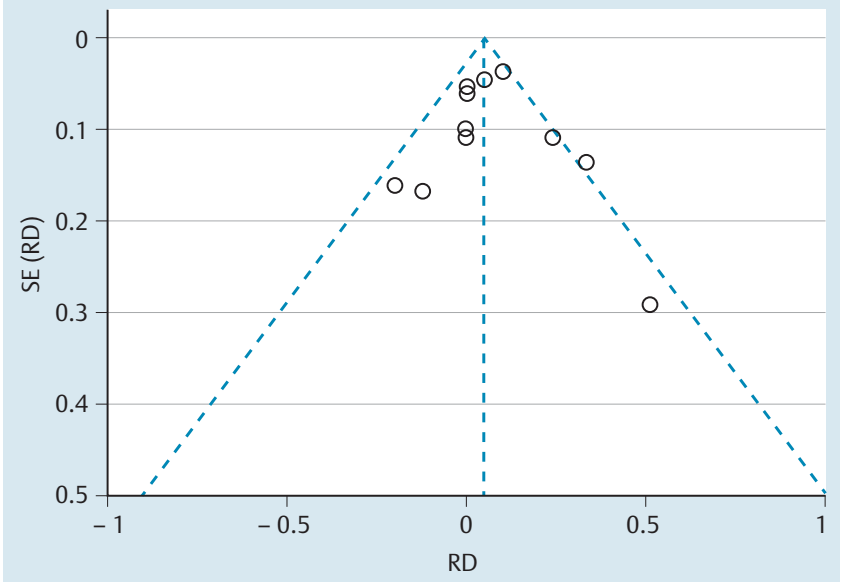

Fig. 7 Recurrence of Zenker's diverticulum: endoscopic and surgical treatment-funnel plot.

ria. All studies were retrospective cohorts. There were no randomized trials. In total, 596 patients were included in the final analysis.

\section{Study characteristics}

The study characteristics of those who were included are summarized in Table1. All 11 studies were retrospective cohorts. The total number of patients, age, gender, and how many were submitted to the endoscopy or surgery groups were observed.

Regarding the treatment approach, the method used in both groups, and the initial size of diverticulum were evaluated. Endoscopic treatment of Zenker's diverticulum includes endoscopic stapling diverticulotomy, $\mathrm{CO}_{2}$ laser, and harmonic scalpel as the most common techniques $[12,14]$. The surgical approach includes cricopharyngeal myotomy and suspension of pouch; cricopharyngeal myotomy and inversion of pouch; cricopharyngeal myotomy and excision of pouch; cricopharyngeal myotomy only; Dolman's procedure and pouch excision only [13].

In terms of outcomes, the data considered were: length of procedure, length of hospitalization, time to diet introduction, compli- cation rates, recurrence rates, improvement in symptoms, conversion rates, costs, and loss to follow-up.

\section{Risk of bias within studies}

All 11 studies that were selected were retrospective and controlled trials (Level of evidence IIB from Oxford Centre for Evidence-based Medicine). The Newcastle-Ottawa Scale was used for quality assessment. The studies selected were all assessed as "acceptable" for the risk of bias, and the detailed information is shown in Table2. As mentioned earlier, the included trials should have a score $\geq 6$, with a total of 9 being the highest possible score (each asterisk symbol in Table 2 represents one point).

\section{Results of individual studies}

The most relevant variables that could be extracted from each study are summarized in Table 3 and include the total number of patients, age, method used in both groups, length of procedure, length of hospitalization, time to introduction of diet, complication rates, recurrence rates, and costs. Table 3 compares the outcomes of the endoscopic versus surgical approach to Zenker's diverticulum.

\section{Synthesis of results}

All 11 studies were reviewed. It was possible to develop a metaanalysis of five outcomes. The other outcomes were not suitable for meta-analysis because of the absence of essential data such as mean and standard deviation or standard error. The outcomes that could be assessed were length of procedure, length of hospitalization, time to diet introduction, rate of complications, and rate of recurrences.

\section{Length of procedure}

The outcome length of procedure was described and contained data that allowed meta-analysis (mean and standard deviation) in three studies, with acceptable heterogeneity (I2=16\%). A statistically significant reduction of operating time was noted favoring endoscopic treatment (standardized mean difference (SMD)$78.06,95 \% \mathrm{CI}-90.63,-65.48)$. Note that "minutes" was used as the unit for the analysis ( Figs. 2,3). 


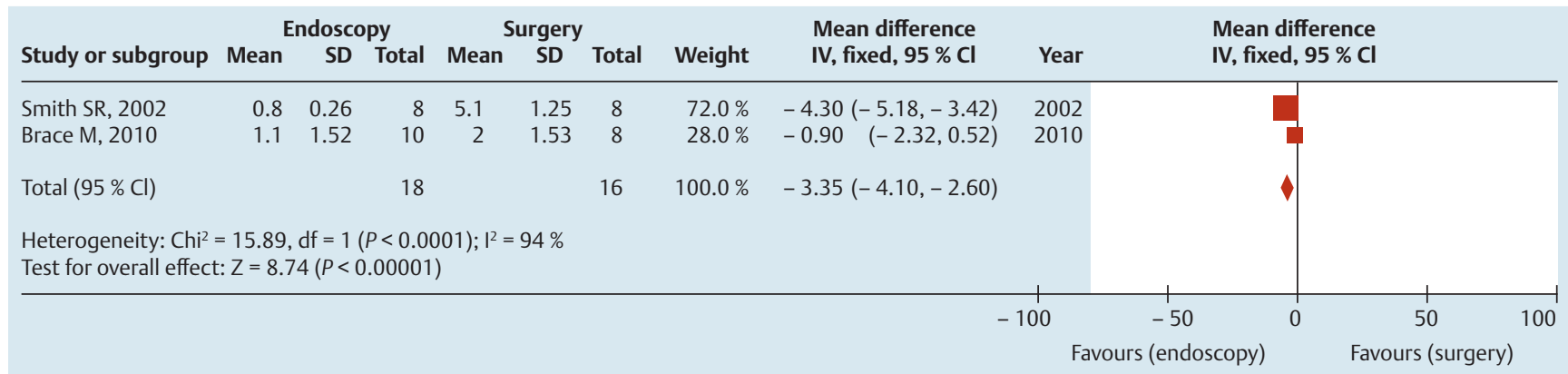

Fig. 8 Time to diet introduction after endoscopic and surgical treatment of Zenker’s diverticulum-forest plot.

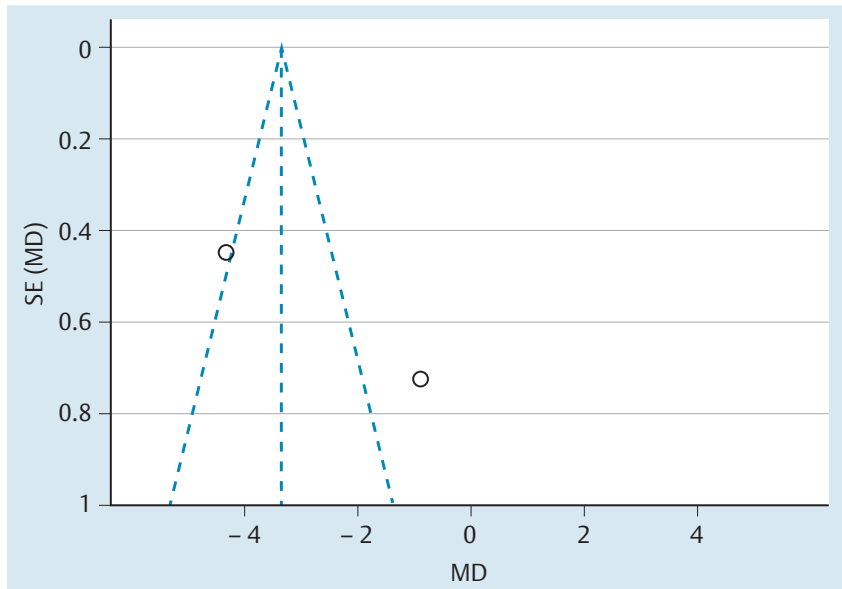

Fig.9 Time to diet introduction after endoscopic and surgical treatment of Zenker's diverticulum-funnel plot.

\section{Length of hospitalization}

The outcome length of hospitalization was suitable for meta-analysis in two studies and they demonstrated homogeneity ( $\mathrm{I} 2=$ $34 \%$ in the funnel plot. A statistically significant reduction in length of hospitalization was observed favoring endoscopic treatment (SMD - 3.72, 95\%CI - 4.49,-2.95). Note that "days" was used as the unit for this analysis ( $\bullet$ Figs. 4,5 ).

\section{Recurrence rates}

All 11 articles had data that were suitable for meta-analysis and had low and acceptable heterogeneity $(I 2=45 \%)$. Meta-analysis showed a statistically significant reduction in the risk of recurrence of symptoms when treatment of Zenker's diverticulum was performed through the surgical approach compared with endoscopic treatment (SMD 0.08, 95\%CI 0.03, 0.13) (৫ Figs. 6, 7).

\section{Diet introduction}

The time to diet introduction after the procedure was found to be suitable for meta-analysis in two studies. Due to a high level of heterogeneity between both articles $(I 2=94 \%)$, the study that had the lowest weight and produced a publication bias was excluded [20]. It was observed that there was a statistically significant reduction in the fasting period for patients who were submitted to endoscopic treatment for Zenker's diverticulum (SMD $4.30,95 \% \mathrm{CI}-5.18,-3.42)$. "Day" was used as the unit during this analysis (० Figs. 8-10).

\section{Complication rates}

All 11 articles had data that allowed meta-analysis of this outcome. The main complications that were described were cervical leak, hoarseness, aspiration pneumonia, chest pain, and perforation of the esophagus. Due to heterogeneity $(\mathrm{I} 2=53 \%)$ promoted by one specific article [18] and identified through the funnel plot, it has been removed from the meta-analysis. After that, the heterogeneity became acceptable ( $\mathrm{I} 2=43 \%$ ). Meta-analysis of complication rates showed a statistically significant reduction in the risk of complications when patients were submitted to endoscopic treatment (SMD-0.09, 95\%CI 0.03, 0.43) in comparison with the surgical approach $(\bullet$ Figs. 11 -13).

\section{Risk of bias across the studies}

All 11 studies presented low indices of heterogeneity detected for the five outcomes that were analyzed (time of operation, length of hospitalization, time to diet introduction, rate of complications, and rate of recurrences).

Time of operation, length of hospitalization, and recurrence rate demonstrated low rates of heterogeneity $(\mathrm{I} 2=16 \%, \mathrm{I} 2=34 \%$, and $\mathrm{I} 2=45 \%$, respectively $)(\bullet$ Figs. $3,5,7)$.

Meta-analysis of time to introduction of diet presented a high level of heterogeneity between two articles $(I 2=94 \%)[20,24]$. One was excluded [20] because of its lowest weight. After that, the concept of heterogeneity became inapplicable for this metaanalysis ( $\bullet$ Fig.9).

Considering complication rates, all 11 articles were suitable for meta-analysis, but the funnel plot demonstrated asymmetry of one of them in comparison with the other 10 studies $(I 2=53 \%)$. That one [18] was removed from the meta-analysis, and then the analysis had an acceptable heterogeneity $(I 2=43 \%)$ (0 Fig. 11).

\section{Discussion \\ $\nabla$}

\section{Summary of evidence}

The treatment of choice for Zenker's diverticulum has historically been surgical. Both open transcervical and endoscopic approaches are associated with complications and potential risks. Nowadays, endoscopic surgery is the first approach [33], but surgery still has its utility in the treatment of Zenker's diverticulum [34]. An incomplete myotomy is associated with a higher degree of symptomatic recurrence [35-39]. Usually, the surgical approach allows diverticulum resection and a complete myotomy, decreasing recurrence. In addition, individual anatomic or disease-related factors, such as upper teeth protrusion or insufficient neck motility hinders endoscopic treatment. So, although not consid- 


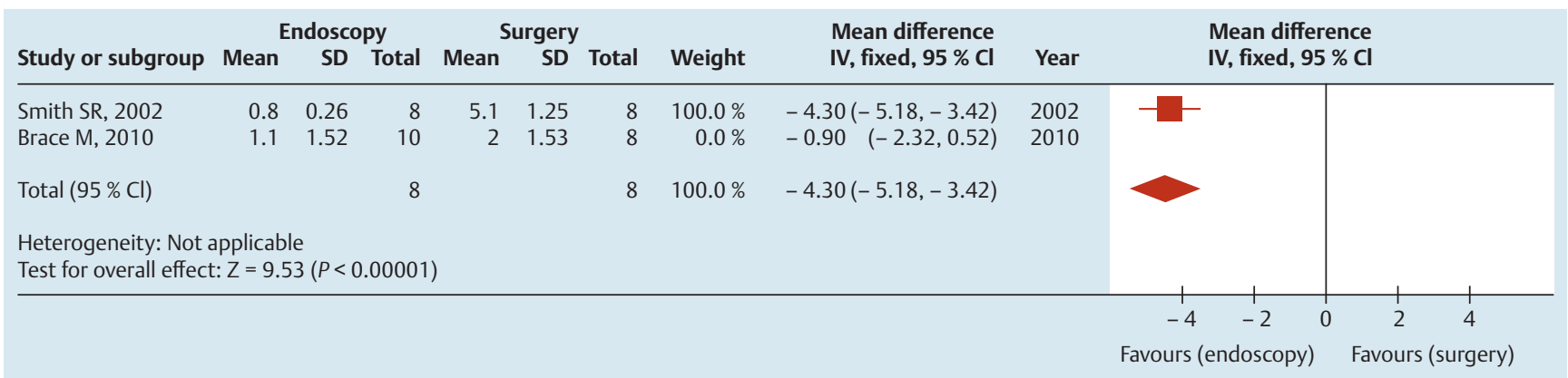

Fig. 10 Time to diet introduction after endoscopic and surgical treatment of Zenker's diverticulum - forest plot (after outlier exclusion).

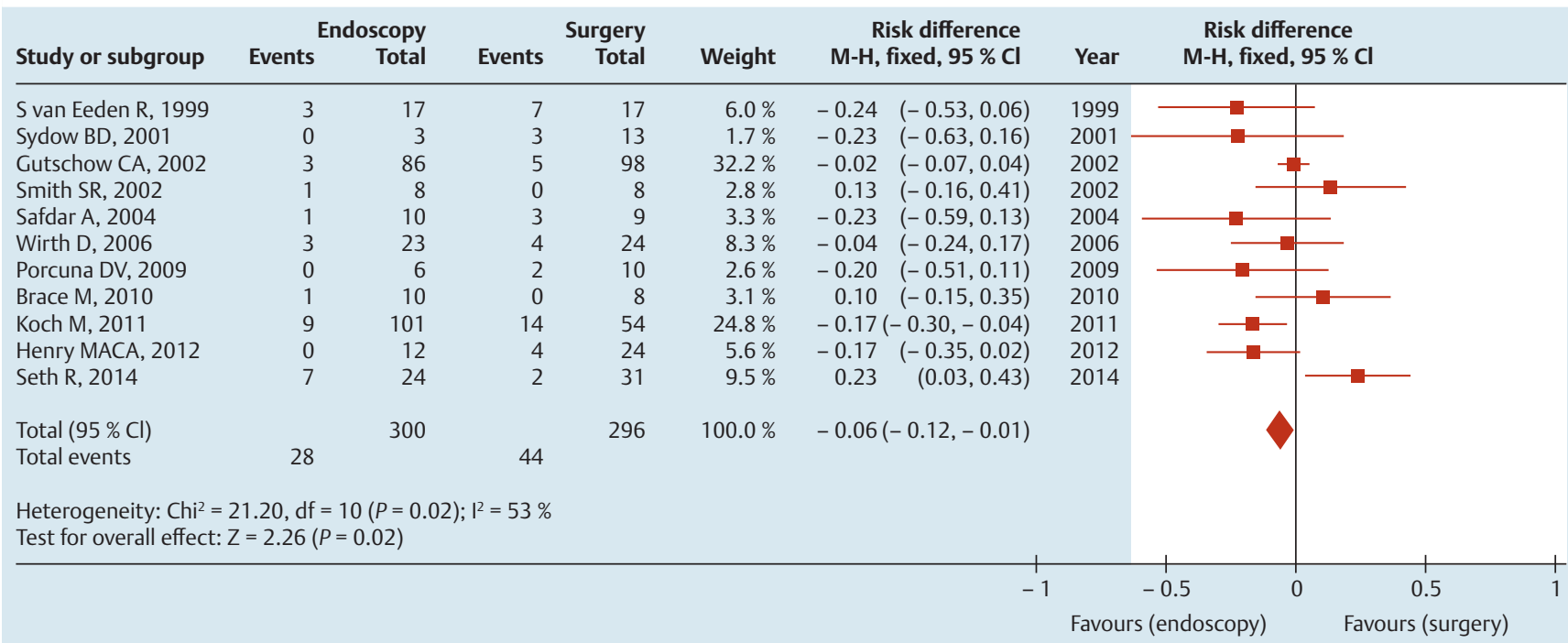

Fig. 11 Rates of complications after endoscopic and surgical treatment-forest plot.

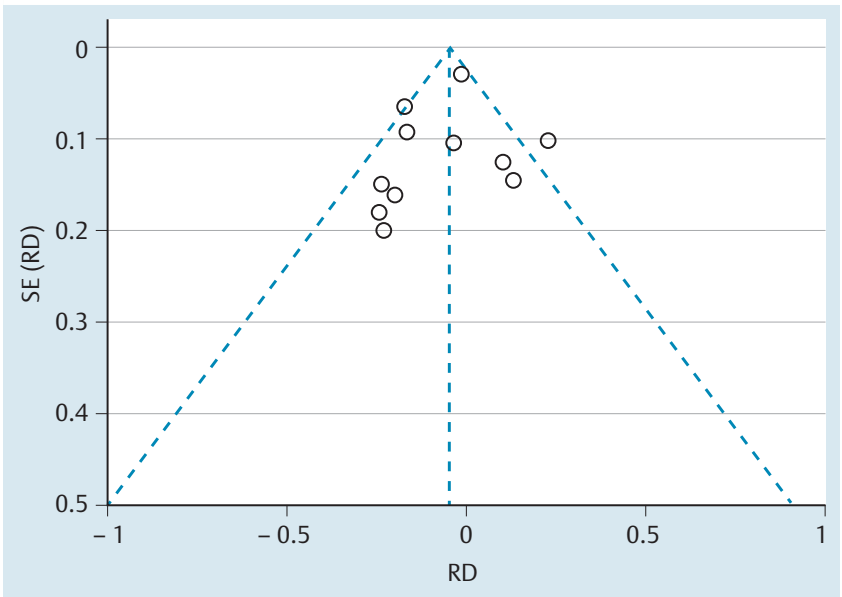

Fig. 12 Rates of complications after endoscopic and surgical treatmentfunnel plot.

ered in this review, some points are important for general consideration. The introduction of the diverticuloscope in the correct position, enabling sufficient and safe exposure of the surgical field is often, in fact, the most difficult and time consuming part of the endoscopic procedure [40]. Exposure of the diverticulum sac, which can be based on clinical conditions (upper teeth protrusion, recessed mandible, narrow mouth opening, cervical spine mobility, position of the larynx, depth of the fundus of the diverticulum sac) and the results of the barium esophagogram (size and anatomy of the pouch, protection by the dorsal esophageal wall) are some adopted criteria for treatment choice [19]. If any major adversity is found, the surgical approach may be considered.

Regarding the size of Zenker's diverticulum, there is no consensus considering different studies [21,22]. A disadvantage of the stapling technique seems to be the incomplete section of the diverticular septum, leaving a remanent sac due to the stapler tip, not reachable by the knife.

In this review, considering all 11 articles, the decision concerning the method to be applied to each patient was variable. Usually, elderly patients tended to be treated with an endoscopic approach, as it has lower rates of complications and a shorter hospital stay [2]. Considering that patients with Zenker's diverticulum usually have many comorbidities, their clinical condition is essential to define the best treatment. Also, physician preferences, training, and standards of practice were important aspects in the final decision $[18,20]$. Seth et al. [18] reported that the patient's wishes should be taken into account. Some authors reported no clinical criteria in choosing the treatment modality $[15,23]$.

Through the systematic review, certain outcomes were found that were not present in all articles and were not suitable for meta-analysis,. The improvement in symptoms was assessed through multiple different questionnaires, usually subjective, which prevented the meta-analysis of this specific outcome.

In summary, patients for open or endoscopic procedures should be selected according to several criteria. Regarding all the nuan- 


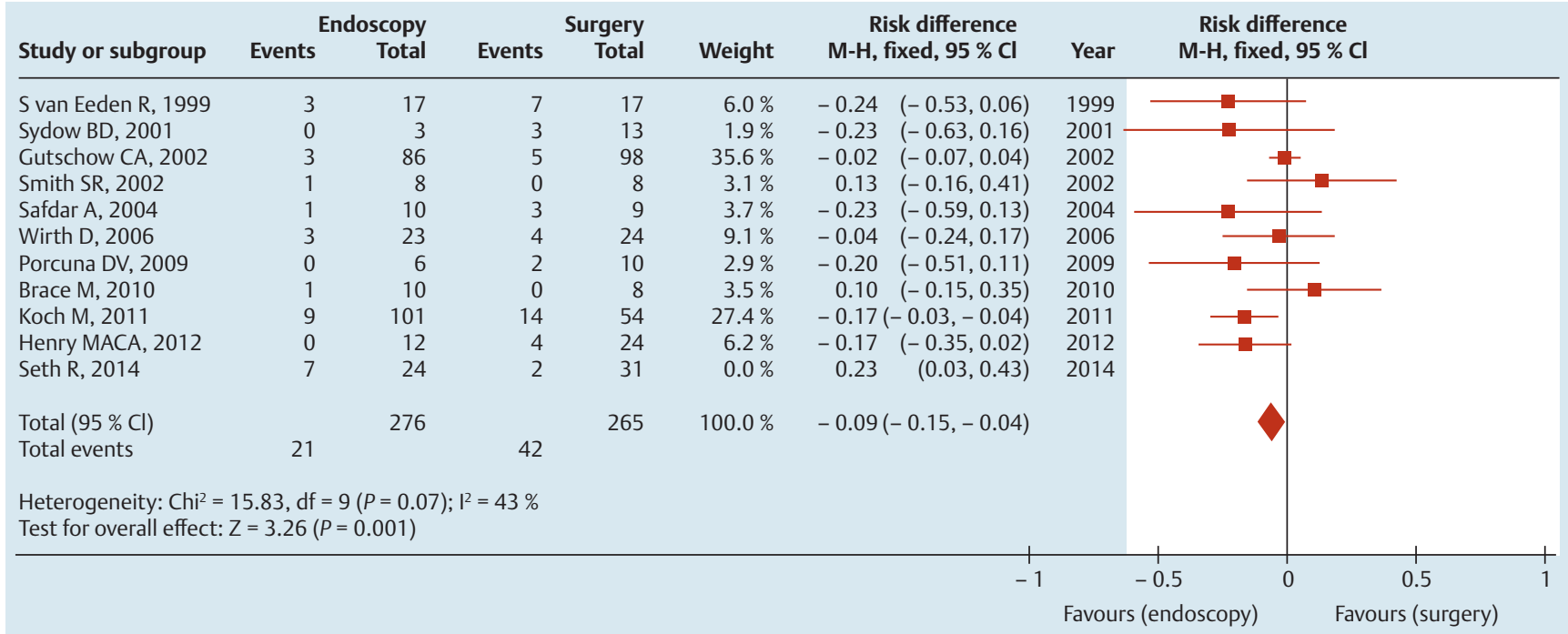

Fig. 13 Rates of complications after endoscopic and surgical treatment-forest plot (after outlier exclusion).

ces of patients with Zenker's diverticulum, the endoscopic approach seems to have better results than surgical management.

\section{Limitations}

In this systematic review, all 11 articles included were retrospective cohorts. In the literature, there were no prospective cohorts or randomized trials comparing both techniques that met the inclusion criteria.

Zenker's diverticulum is a rare disease and typical of an elderly population, which may confound cases of complications or even death due to other health problems.

The decision to allocate patients to the groups could be biased by the age of the patient, diverticulum size, and anatomic variation that induces the patient to be allocated to one specific group.

Analysis of symptom improvement was evaluated in a variable manner in each article and was associated with a large loss at follow-up, which constrained the statistical analysis.

\section{Conclusions}

Compared with a surgical approach, endoscopic treatment appears to result in a shorter length of procedure and hospitalization, earlier diet introduction, and lower rates of complications, but in higher rates of symptom recurrence.

\section{Competing interests: None}

\section{References}

1 Zenker FA, von Ziemssen $H$. Krankenheiten des oesophagus. In: von Ziemssen $\mathrm{H}$, ed. Handbuch der Speziellen Pathologie und Therapie. Leipzig: FCW Vogel; 1877: 1-87

2 Henry MA, Lerco MM, Tagliarini JV et al. Pharyngoesophageal diverticulum: evaluation of treatment results. Rev Col Bras Cir 2013; 40: $104-$ 109

3 Sabiston DC Jr. Textbook of surgery: The biological basis of modern surgical practice. Philadelphia, PA: WB Saunders; 1997

4 Bizzotto A, Iacopini $F$, Landi $R$ et al. Zenker's diverticulum: exploring treatment options. Acta Otorhinolaryngol Ital 2013; 33: 219-229

5 Peters JH, Mason R. The physiopathological basis for Zenker's diverticulum. Chirurg 1999; 70: 741 - 746

6 Fasano NC, Levine MS, Rubesin SE et al. Epiphrenic diverticulum: clinical and radiographic findings in 27 patients. Dysphagia 2003; 18: 9 15
7 Klaus A, Hinder RA, Swain J et al. Management of epiphrenic diverticula. J Gastrointest Surg 2003; 7: 906-911

8 Mosher HP. Webs and pouches of the esophagus, their diagnosis and treatment. Surg Gynecol Obstet 1917; 25: 175-187

9 Dohlman G, Mattsson 0 . The endoscopic operation for hypopharyngeal diverticula: a roentgencinematographic study. AMA Arch Otolaryngol 1960; 71: 744-752

10 Benjamin B, Innocenti M. Laser treatment of pharyngeal pouch. Aust N Z J Surg 1991; 61: 909-913

11 Knegt PP, de Jong PC, van der Schans EJ. Endoscopic treatment of the hypopharyngeal diverticulum with the CO2 laser. Endoscopy 1985; 17: 205-206

12 Von Doersten PG, Byl FM. Endoscopic Zenker's diverticulotomy (Dohlman procedure): forty cases reviewed. Otolaryngol Head Neck Surg 1997; 116: 209-212

13 Nyrop M, Svendstrup F, Jørgensen KE. Endoscopic CO2 laser therapy of Zenker's diverticulum - experience from 61 patients. Acta Otolaryngol Suppl 2000; 543: 232-234

14 Collard JM, Otte JB, Kestens PJ. Endoscopic stapling technique of esophagodiverticulostomy for Zenker's diverticulum. Ann Thorac Surg 1993; 56: 573-576

15 van Eeden S, Lloyd RV, Tranter RM. Comparison of the endoscopic stapling technique with more established procedures for pharyngeal pouches: results and patient satisfaction survey. J Laryngol Otol 1999; 113: $237-240$

16 Moher D, Liberati A, Tetzlaff J et al. Preferred reporting items for systematic reviews and meta-analyses: The PRISMA statement. BM] 2009; 339: b2535

17 Wells GA, Shea B, O'Connell D et al. The Newcastle-Ottawa Scale (NOS) for Assessing the Quality of Nonrandomised Studies in Meta-analysis. 01. 02 2009: http://www.ohri.ca/programs/clinical_epidemiology/oxford.htm

18 Seth R, Rajasekaran K, Lee WT et al. Patient reported outcomes in endoscopic and open transcervical treatment for Zenker's diverticulum. Laryngoscope 2014; 124: 119-125

19 Koch M, Mantsopoulos K, Velegrakis S et al. Endoscopic laser-assisted diverticulotomy versus open surgical approach in the treatment of Zenker's diverticulum. Laryngoscope 2011; 121: 2090-2094

20 Brace M, Taylor SM, Trites JR et al. Endoscopic stapling versus external transcervical approach for the treatment of Zenker diverticulum. J Otolaryngol Head Neck Surg 2010; 39: $102-106$

21 Virós Porcuna D, Zarraonandía Andraca I, León Vintró X et al. Combined endoscopic treatment for Zenker's diverticulum versus open approach; review of our experience. Acta Otorrinolaringol Esp 2009; 60: $396-401$

22 Wirth D, Kern B, Guenin MO et al. Outcome and quality of life after open surgery versus endoscopic stapler-assisted esophagodiverticulostomy for Zenker's diverticulum. Dis Esophagus 2006; 19: 294-298 
23 Safdar A, Curran A, Timon CV. Endoscopic stapling vs conventional methods of surgery for pharyngeal pouches: results, benefits and modifications. Ir Med J 2004; 97: 75-76

24 Smith SR, Genden EM, Urken ML. Endoscopic stapling technique for the treatment of Zenker diverticulum vs standard open-neck technique: a direct comparison and charge analysis. Arch Otolaryngol Head Neck Surg 2002; 128: 141 - 144

25 Gutschow CA, Hamoir M, Rombaux P et al. Management of pharyngoesophageal (Zenker's) diverticulum: which technique? Ann Thorac Surg 2002; 74: 1677-1682; discussion 1682-1683

26 Sydow BD, Levine MS, Rubesin SE et al. Radiographic findings and complications after surgical or endoscopic repair of Zenker's diverticulum in 16 patients. AJR Am J Roentgenol 2001; 177: 1067-1071

27 Higgins JP, Thompson SG. Quantifying heterogeneity in a meta-analysis. Stat Med 2002; 21: 1539-1558

28 Hedges LV, Pigott TD. The power of statistical tests for moderators in meta-analysis. Psychol Methods 2004; 9: 426-445

29 Sutton AJ, Abrams KR, Jones DR et al. Methods for meta-analysis in medical research. New York, Chichester: Wiley; 2000

30 Huedo-Medina TB, Sánchez-Meca J, Marín-Martínez F et al. Assessing heterogeneity in meta-analysis: Q statistic or I2 index? Psychol Methods 2006; 11: 193-206

31 Liu IM, Agresti A. Mantel-Haenszel-type inference for cumulative odds ratios with a stratified ordinal response. Bio-metrics 1996; 52: 1223 1234
32 The Nordic Cochrane Centre. The Cochrane Collaboration. Review Manager (RevMan) [Computer program]. Version 5.3 Copenhagen: The Nordic Cochrane Centre, The Cochrane Collaboration; 2014

33 Hondo FY, Maluf-Filho F, Giordano-Nappi JH et al. Endoscopic treatment of Zenker's diverticulum by harmonic scalpel. Gastrointest Endosc 2011; 74: 666-671

34 Brombart $M$. Le diverticule pharyngo-oesophagien de Zenker. Considerations pathogenetiques. J Belg Radiol 1953; 76: 128

35 Chang CW, Burkey BB, Netterville JL et al. Carbon dioxide laser endoscopic diverticulotomy versus open diverticulectomy for Zenker's diverticulum. Laryngoscope 2004; 114: 519-527

36 Visosky AM, Parke RB, Donovan DT. Endoscopic management of Zenker's diverticulum: factors predictive of success or failure. Ann Otol Rhinol Laryngol 2008; 117: 531-537

37 Krespi Y, Kacker A, Remacle M. Endoscopic treatment of Zenker's diverticulum using CO2 laser. Otolaryngol Head Neck Surg 2002; 127: 309 314

38 Rizzetto C, Zaninotto G, Costantini M et al. Zenker's diverticula: feasibility of a tailored approach based on diverticulum size. J Gastrointest Surg 2008; 12: 2057-2064

39 Bloom JD, Bleier BS, Mirza N et al. Factors predicting endoscopic exposure of Zenker's diverticulum. Ann Otol Rhinol Laryngol 2010; 119: $736-741$

40 Saetti R, Silvestrini $M$, Peracchia A et al. Endoscopic stapler-assisted Zenker's diverticulotomy: which is the best operative facility? Head Neck 2006; 28: 1084-1089 